\title{
Prenatal diet and childhood ADHD: exploring the potential role of IGF2 methylation
}

\section{"Evidence for diet-induced epigenetic effects may also highlight nutrition as an important modifiable intervention target."}

First draft submitted: 29 September 2016; Accepted for publication: 6 October 2016; Published online: 18 November 2016

Keywords: attention-deficit hyperactivity $\bullet$ DNA methylation $\bullet$ early-onset conduct problems - epidemiology $\bullet / G F 2 \bullet$ prenatal diet $\bullet$ prenatal healthy $\bullet$ unhealthy diet

Unhealthy diet during pregnancy is a risk factor for a wide range of negative health and psychiatric outcomes [1]. For example, high-sugar and fat diets associate not only with increased risk for noncommunicable diseases [2], such as diabetes and obesity, but also for neurodevelopmental disorders, such as attention-deficit hyperactivity disorder (ADHD) and conduct problems (CP) [3]. In the nutritional field, epigenetics is an important area of investigation, as nutrients and bioactive compounds can alter the expression of genes at the transcriptional level [4]. Because epigenetic modifications, such as DNA methylation, can be passed on during cell division and result in long-term phenotypic changes [5], they also provide a framework for understanding the biological mechanisms through which pre- and post-natal environmental exposures may influence disease vulnerability $[6,7]$.

In a recent paper [8], we examined how exposure to unhealthy fats (e.g., vegetable oils in fast foods) and sugars (e.g., sweets) might associate with ADHD symptoms in children who follow an early onset (age $\leq 10$ years; $\mathrm{n}=83)$ versus low $(\mathrm{n}=81)$ trajectory of $C P$, via DNA methylation of the IGF2 gene. We focused on IGF2 due to its relevance in metabolic function [9], placental and fetal growth [10] and the development of brain regions implicated in ADHD [11-13]. Data were drawn from the Accessible Resource for Integrated Epigenomics Studies [14], a sub- sample of the Avon Longitudinal Study of Parents and Children, which includes: firstly, maternal reports of diet (32-week gestation, age 3 and 7 years); secondly, peripheral measures of DNA methylation (Illumina $450 \mathrm{k}$ ) at birth and age 7 years $(n=671,49 \%$ male) and thirdly, repeated assessments of psychiatric symptomatology, including CP (ages 4-13 years) and ADHD (ages 7-13 years). Below, we summarize our key findings before discussing limitations and future directions.

\section{Key findings}

Prenatal unhealthy diet \& neonatal IGF2 methylation

Firstly, we found that a maternal unhealthy diet during pregnancy prospectively associated with higher IGF2 methylation at birth across all (i.e., both CP and typically developing) children, even after adjusting for a range of inter-related risk factors (e.g., maternal smoking, psychosocial and contextual risks). This finding is consistent with previous research reporting an association between variation in offspring IGF2 methylation and prenatal dietary exposures, including periconceptional dietary supplementation (e.g., folic acid [15,16]; docosahexaenoic acid [17]), severe caloric restriction resulting from prenatal famine exposure [18], maternal obesity and BMI [17,19], as well as animal research on high-fat diet exposure [20]. The use of a prospective design additionally enabled us to examine longitudinal inter-
Epigenomics

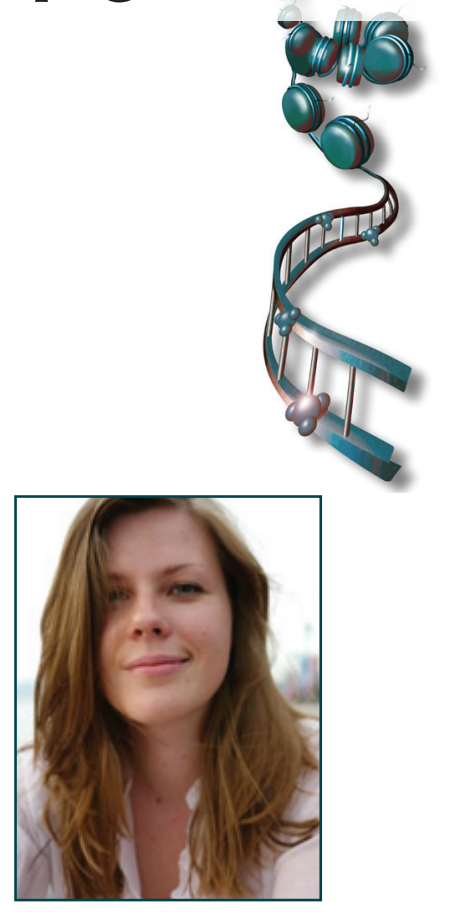

Charlotte AM Cecil

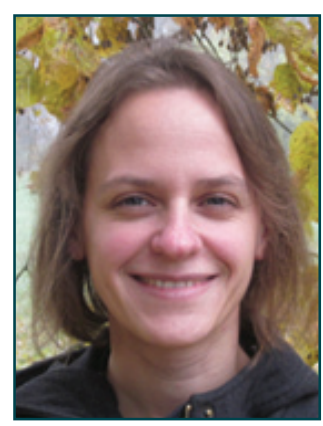

Esther Walton

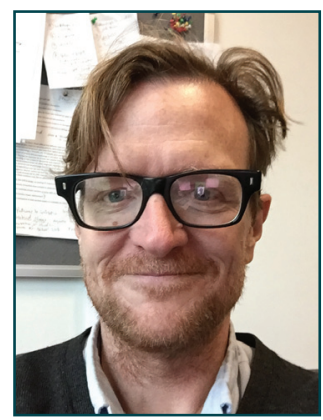

Edward D Barker

Author for correspondence: Tel.: +44 (0)2078480389 ted.barker@kcl.ac.uk For a full list of affiliations, please see page 1576

Future : Medicine part of 
relationships between unhealthy diet and IGF2 methylation, spanning gestation to mid-childhood. We found that, firstly, the association between diet and IGF2 methylation was specific to pregnancy (i.e., not observed postnatally) and secondly, whereas unhealthy diet showed high stability over time (i.e., what mothers ate in pregnancy related to what their children ate in childhood), IGF2 methylation did not. While these findings do not permit causal inference (see limitations), they do point to gestation as a potentially critical developmental window for diet-induced changes in IGF2 methylation, and suggest that IGF2 methylation levels may be temporally dynamic.

IGF2 methylation at birth \& ADHD symptoms in childhood

Secondly, we found that IGF2 methylation at birth prospectively associated with higher ADHD symptoms in childhood (age 7-13 years), but that this association was specific to $\mathrm{CP}$ children. Interestingly, this specificity was not due to differences in dietary exposure, as both groups showed comparable levels of prenatal unhealthy diet as well as IGF2 methylation at birth. Why would such a developmental risk pathway be specific to $\mathrm{CP}$ children? We know from a large body of literature that $\mathrm{CP}$ and ADHD are not only highly co-morbid, but that this co-occurrence is underpinned by greater genetic heritability and more severe environmental risk exposure, compared with either $\mathrm{CP}$ or ADHD alone [21,22]. Consequently, it is possible that, for CP children, the association between IGF2 methylation and ADHD may be compounded by unmeasured genetic influences (e.g., variability in IGF2, related imprinted loci, such as H19, and/or broader polygenic effects) as well as environmental factors beyond those controlled for in the analyses (e.g., air pollutants [23]).

\section{IGF2 methylation as a potential mediating mechanism}

Thirdly, we found that among CP children, IGF2 methylation at birth mediated the effect of prenatal diet on ADHD symptomatology. This finding supports previous data from animal studies documenting an indirect pathway linking prenatal diet and postnatal outcomes, via IGF2 methylation. For example, Claycombe et al. [20] reported that in male rats, combined exposure to intra-uterine undernourishment and post-weaning high-fat diet associated with variation in IGF2 methylation and expression, which in turn related to lower insulin sensitivity and higher adipose tissue growth. Here, we extend findings by implicating IGF2 methylation as a potential mechanism not only mediating vulnerability to physical/metabolic outcomes but also for ADHD risk, a neurodevelopmental psychiatric disorder. Of note, we found that mediation was specific to ADHD symptoms and did not extend to other cooccurring symptoms (e.g., anxiety, depression) among $\mathrm{CP}$ children. Together, these findings are in line with the developmental origins of health and disease [7] and latent vulnerability [24] hypotheses, whereby early-risk exposure may alter biological systems in a way that may increase long-term vulnerability to physical and psychiatric disease.

\section{Limitations \& future perspective}

Conclusions drawn from our study are limited in a few important ways. Firstly, ADHD and CP are complex, multi-determined psychiatric phenotypes. As such, other factors, such as genetic and environmental influences (beyond diet), are likely to be important. Moreover, the IGF2 gene is located in a highly dynamic region that includes multiple transcripts from alternative promoters, relates to different biological functions and is differentially expressed across tissues and developmental periods, so that a more comprehensive epigenetic investigation in this gene will be required (e.g., contribution of histone modifications and miRNAs).

Secondly, we examined ADHD as a global construct. However, ADHD comprises of subdimensions of inattention, hyperactivity and impulsivity, and it will be of interest in future to establish whether effects may be general or domain-specific [25]. Thirdly, although the use of peripheral tissue samples hold potential for the identification of exposure/risk biomarkers, the extent to which findings may reflect methylation changes in the brain is unclear. In fact, a recent study [26] found that most DNA methylation markers in peripheral blood do not reliably predict brain DNA methylation status, making inferences on brain-relevant processes difficult. As such, it will be important to establish to what extent peripheral levels of IGF2 methylation relate to in vivo structural/functional neural markers of ADHD.

Fourthly, more work will be needed to trace the specific biological pathways through which the observed effects manifest. The present findings allow only limited conclusions as to, firstly, how exactly diet affects IGF2 methylation; secondly, why this effect is only observed prenatally and thirdly, why associations with ADHD are observed only in children with co-occurring $\mathrm{CP}$. Addressing these questions will require the integration of intermediary variables, such as metabolites of nutrition, fetal growth trajectories (e.g., via sonar doppler; [27]), transcriptomic data, early indicators of neurodevelopment and in vivo brain-imaging measures. The use of more comprehensive, repeated assessments of prenatal diet will also enable us to trace 
dietary effects on IGF2 methylation from early-tolate gestation, as well as examining the role of other important nutrients (e.g., folic acid). Furthermore, the inclusion of genetic and environmental moderators may help to shed light on the specificity of ADHD risk to $\mathrm{CP}$ children (e.g., via molecular designs to study single-gene effects; or behavioral genetic designs to investigate diet interaction with genetic heritability as a whole).

Finally, because our study was based on correlational data, we were not able to establish causality. This will require the use of a multimethod approach for strengthening causal inference, including the implementation of (two-step) mendelian randomization [28], negative control (e.g., using paternal prenatal diet effects as a negative control to maternal effects) and cross-species designs.

\section{Implications \& translational potential}

Data from our study and others suggest that IGF2 methylation may be sensitive to diet-related exposures - particularly during pregnancy - and mediate vulnerability for negative developmental outcomes, such as ADHD risk. Although promising, this evidence is currently preliminary and in need of replication. Consequently, findings should be interpreted with caution and considered more as well-grounded hypotheses for further investigation. Bearing this in mind, there are

\section{References}

1 Barouki R, Gluckman PD, Grandjean P, Hanson M, Heindel JJ. Developmental origins of non-communicable disease: implications for research and public health. Environ. Health 11(1), 1 (2012).

2 Waxman A, Norum KR. Why a global strategy on diet, physical activity and health? The growing burden of noncommunicable diseases. Pub. Health Nutr. 7(03), 381-383 (2004).

3 American Psychiatric Association. Diagnostic and Statistical Manual of Mental Disorders (DSM- $\left.5^{\circledR}\right)$. American Psychiatric Association, VA, USA (2013).

4 Choi S-W, Friso S. Epigenetics: a new bridge between nutrition and health. Adv. Nutr. 1(1), 8-16 (2010).

5 Jirtle RL, Skinner MK. Environmental epigenomics and disease susceptibility. Nat. Rev. Genet. 8, 253-262 (2007).

6 Mill J, Heijmans BT. From promises to practical strategies in epigenetic epidemiology. Nat. Rev. Genet. 14(8), 585-594 (2013).

7 Barker DJ. The origins of the developmental origins theory. J. Intern. Med. 261(5), 412-417 (2007).

8 Rijlaarsdam J, Cecil CA, Walton E et al. Prenatal unhealthy diet, insulin-like growth factor 2 gene (IGF2) methylation, and attention deficit hyperactivity disorder symptoms in youth with early-onset conduct problems. J. Child. Psychol. a number of ways in which findings may be used in future to inform policy and practice. In the first place, findings may refine existing models of how risk exposures, such as an unhealthy diet, become biologically embedded. Longitudinal modeling of environmental and epigenetic data may also be used to pinpoint specific windows of biological vulnerability (e.g., prenatal period) that may benefit most from preventive action. Evidence for diet-induced epigenetic effects may also highlight nutrition as an important modifiable intervention target. On the longer term, developments in knowledge, methodology and research designs will offer exciting opportunities for delineating the role of IGF2 methylation, the neurodevelopment, as well as testing its potential clinical utility as an exposure indicator, disease biomarker and therapeutic target.

\section{Financial \& competing interests disclosure}

Preparation of this manuscript was supported by the Economic and Social Research Council (CAM Cecil; grant ref: ES/N001273/1) and National Institute of Child and Human Development grant (ED Barker; R01HD068437). The authors have no other relevant affiliations or financial involvement with any organization or entity with a financial interest in or financial conflict with the subject matter or materials discussed in the manuscript apart from those disclosed.

No writing assistance was utilized in the production of this manuscript.

Psychiatry doi: 10.1111/jcpp.12589 (2016) (Epub ahead of print).

9 Ukkola O, Sun G, Bouchard C. Insulin-like growth factor 2 (IGF2) and IGF-binding protein 1 (IGFBP1) gene variants are associated with overfeeding-induced metabolic changes. Diabetologia 44(12), 2231-2236 (2001).

10 Constância M, Hemberger M, Hughes J et al. Placentalspecific IGF-II is a major modulator of placental and fetal growth. Nature 417(6892), 945-948 (2002).

11 Pidsley R, Dempster E, Troakes C, Al-Sarraj S, Mill J. Epigenetic and genetic variation at the IGF2/H19 imprinting control region on $11 \mathrm{p} 15.5$ is associated with cerebellum weight. Epigenetics 7(2), 155-163 (2012).

12 Castellanos FX, Lee PP, Sharp W et al. Developmental trajectories of brain volume abnormalities in children and adolescents with attention-deficit/hyperactivity disorder. JAMA 288(14), 1740-1748 (2002).

13 Plessen KJ, Bansal R, Zhu $\mathrm{H}$ et al. Hippocampus and amygdala morphology in attention-deficit/hyperactivity disorder. Arch. Gen. Psychiatr. 63(7), 795-807 (2006).

14 Relton CL, Gaunt T, McArdle W et al. Data resource profile: Accessible Resource for Integrated Epigenomic Studies (ARIES). Int. J. Epidemiol. 44(4), 1181-1190 (2015).

15 Steegers-Theunissen RP, Obermann-Borst SA, Kremer D et al. Periconceptional maternal folic acid use of $400 \mu \mathrm{g}$ per 
day is related to increased methylation of the $I G F 2$ gene in the very young child. PLoS ONE 4(11), e7845 (2009).

16 Hoyo C, Murtha AP, Schildkraut JM et al. Methylation variation at $I G F 2$ differentially methylated regions and maternal folic acid use before and during pregnancy. Epigenetics 6(7), 928-936 (2011).

17 Lee H-S, Barraza-Villarreal A, Biessy C et al. Dietary supplementation with polyunsaturated fatty acid during pregnancy modulates DNA methylation at IGF2/H19 imprinted genes and growth of infants. Physiol. Genom. 46(23), 851-857 (2014).

18 Heijmans BT, Tobi EW, Stein AD et al. Persistent epigenetic differences associated with prenatal exposure to famine in humans. Proc. Natl Acad. Sci. USA 105(44), 17046-17049 (2008).

19 Soubry A, Murphy S, Wang F et al. Newborns of obese parents have altered DNA methylation patterns at imprinted genes. Int. J. Obes. (Lond.) 39(4), 650-657 (2013).

20 Claycombe KJ, Uthus EO, Roemmich JN, Johnson LK, Johnson WT. Prenatal low-protein and postnatal high-fat diets induce rapid adipose tissue growth by inducing Igf2 expression in Sprague Dawley rat offspring. J. Nutr. 143(10), 1533-1539 (2013).

21 Thapar A, Harrington R, McGuffin P. Examining the comorbidity of ADHD-related behaviours and conduct problems using a twin study design. Br. J. Psychiatry 179(3), 224-229 (2001).

\section{Affiliations}

\section{Charlotte AM Cecil}

Department of Psychology, Institute of Psychiatry, Psychology

\& Neuroscience, King's College London, London, UK

\section{Esther Walton}

Department of Psychology, Institute of Psychiatry, Psychology

\& Neuroscience, King's College London, London, UK

and

MRC Integrative Epidemiology Unit, School of Social \& Community

Medicine, University of Bristol, Bristol, UK
22 Hamshere ML, Langley K, Martin J et al. High loading of polygenic risk for ADHD in children with comorbid aggression. Am. J. Psychiatry 170 (8), 909-916 (2013).

23 Fluegge K. Does environmental exposure to the greenhouse gas, $\mathrm{N}_{2} \mathrm{O}$, contribute to etiological factors in neurodevelopmental disorders? A mini-review of the evidence. Environ. Toxicol. Pharmacol. 47, 6-18 (2016).

24 McCrory EJ, Viding E. The theory of latent vulnerability: reconceptualizing the link between childhood maltreatment and psychiatric disorder. Dev. Psychopathol. 27(2), 493-505 (2015).

25 Larsson H, Dilshad R, Lichtenstein P, Barker ED. Developmental trajectories of DSM-IV symptoms of attention-deficit/hyperactivity disorder: genetic effects, family risk and associated psychopathology. J. Child. Psychol. Psychiatry 52(9), 954-963 (2011).

26 Walton E, Hass J, Liu J et al. Correspondence of DNA methylation between blood and brain tissue and its application to schizophrenia research. Schizophr. Bull. 42(2), 406-414 (2015).

27 Barker ED, McAuliffe FM, Alderdice F et al. The role of growth trajectories in classifying fetal growth restriction. Obstet. Gynecol. 122(2, Part 1), 248-254 (2013).

28 Relton CL, Davey Smith G. Two-step epigenetic Mendelian randomization: a strategy for establishing the causal role of epigenetic processes in pathways to disease. Int. J. Epidemiol. 41(1), 161-176 (2012).

\section{Edward D Barker}

Department of Psychology, Institute of Psychiatry, Psychology \& Neuroscience, King's College London, London, UK 